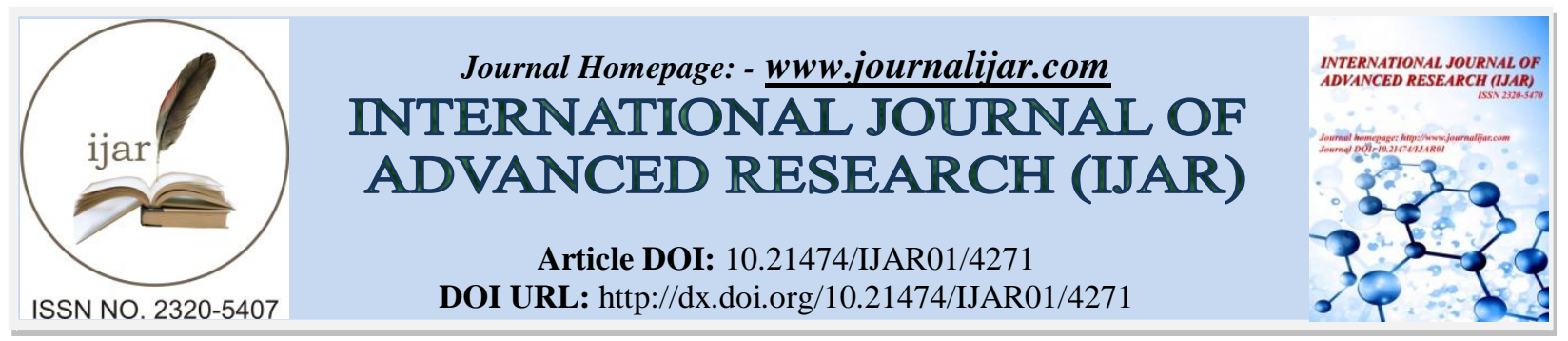

RESEARCH ARTICLE

\title{
STIFFSHAFT DYNAMIC LOADING OF THE MANUAL MOTORIZED ROTATIONAL MOWING MACHINE AT THE MOMENT OF CLIMBING.
}

\author{
M. Mamuladze ${ }^{1}$, M. Lejava ${ }^{2}$ and F. Abuselidze ${ }^{3}$. \\ 1. Associated Professor at Batumi Shota Rustaveli State University. \\ 2. Associated Professor at Batumi State Maritime Academ. \\ 3. Associated Professor at Tbel Abuseridze Teaching University.
}

\section{Manuscript Info}

Manuscript History

Received: 27 March 2017

Final Accepted: 30 April 2017

Published: May 2017

Key words:-

mowing machine, engine,

stiffshaft, cutting device

\begin{abstract}
following the example of in the mountain regions the restoration perspective of the agrarian process showed that animal industry stays one of the priorities study that is determined by manufacturing the animal industry. Coming from the given above the problematic issue for the small farms and farmer industry stays preparation problem of the rough food for the mining environment where the large power machines are impossible to be used and still today the partial food production is accomplished manually. Coming from the given above must be improved and innovated such small mechanized machines which are able to solve the given problem. In the scientific work is represented dynamic loading of small powered manual motorized mowing machine at the moment of climbing which is based largely on increased movement time, workers work and security for the small outlined areas and mining conditions for processing the mowing processes economically and quickly.
\end{abstract}

Copy Right, IJAR, 2017,. All rights reserved.

\section{Introduction:-}

A study was undertaken to investigate the influence of shaft stiffness on the deflection and the positioning of the club face during a golf swing. To accomplish this task, a theoretical model was developed that calculated deflections of golf shafts during the swing. Applying moment-curvature in relation to a shaft that had the bending stiffness, a differential equation and the boundary conditions that characterized its behavior over the duration of the swing were generated. Incorporated into the bending moment-distribution are forces and torques acting at each end of the shaft. These loads were calculated using an inverse dynamics problem of the golf club during the swing. A motion capture system was utilized to collect the necessary data. Solutions to the equations of motion for the shaft were calculated numerically. The results have been presented as deflections along the shaft as functions of time. Bending deflections were determined in the plane of the club face and normal to this plane were calculated. (Ken Ohta, Yuji Ohgi, Kazuhiro Shibuya, 1999).

The dynamic stability of a system composed of driving and driven shafts connected by a universal joint is investigated. Due to the characteristics of the joint, even if the driving shaft experiences constant torque and rotational speed, the driven shaft experiences fluctuating rotational speed, bending moments and torque. These are 
sources of potential parametric, forced and flutter type instabilities. The focus of this work is on the lateral instabilities of the driven shaft. Two distinct models are developed, namely, a rigid body model (linear and nonlinear) and a flexible model (linear). The driven shaft is taken to be pinned at the joint end and to be resting on a compliant damped bearing at the other end. Both models lead to sets of differential equations with time dependent coefficients. For both rigid (linear and non-linear) and flexible models, flutter instabilities were found but occurred outside the practical range of operation (rotational speed and torque) for lightly damped systems. Parametric instability charts were obtained by using the monodromy matrix technique for both rigid and flexible linear models. The transmitted bending moments were found to cause strong parametric instabilities in the system. By comparing the results from the two linear models, it is shown that the inclusion of flexibility leads to new zones of instability, not predicted by previous models. These zones, depending on the physical parameters of the system, can occur for practical conditions of operation. Using direct numerical integration for a few sets of specific parameter values, forced resonances were found when the rotational speed reached a value equal to a natural frequency of the system divided by two.( A.J Mazzei Jr, A. Argento, R.A. Scott,1999).

Efficient C0 -compatible finite elements for the modelling of rotor-bearing systems. The proposed linearly tapered elements, which have a variable number of nodal points, are simple and attractive from a cost viewpoint. They include the effects of translational and rotatory inertia, gyroscopic moments, internal viscous and hysteretic damping, shear deformations, and mass eccentricity. Developed from the weak formulation associated with the differential equations governing the transverse dynamic behavior of rotors, these elements show a convergence pattern similar to the one obtained with conventional $\mathrm{C} 1$-compatible shaft elements. Numerical examples are provided, which compare the proposed approach to the $\mathrm{C} 1$-formulation or to previously published results.( $\mathrm{T}$. $\mathrm{C}$. Gmür, J. D. Rodrigues, 1991)

A new multi-coordinate dynamic model of a hypoid geared rotor system considering the propeller shaft bending effect is formulated. The bending flexibility of propeller shaft is modeled as a lumped parameter set applying the component mode synthesis. The pinion and gear are modeled as flexibly coupled rigid bodies with backlash nonlinearity and time-varying mesh coupling. The kinematic transmission error is assumed to be the main excitation. The solution is obtained by employing the explicit Runge-Kutta integration method with adaptive time step, which yields the dynamic mesh force and also the vibratory response of the gear bodies in time domain. The frequency response functions are then determined by computing the root-mean-square of the time domain solutions at each frequency point. The analyses show that the effect of propeller shaft bending flexibility and damping on the geared rotor system dynamics are found to be mode shape dependent. It is further observed that increasing the damping of propeller shaft can effectively reduce the dynamic mesh force. This indicates that the damping treatment on propeller shaft can be a potential application to reduce the gearbox vibration and noise response. (Yang, T. C. Lim, 2015)

\section{Materials and Method:-}

Motor or electro engine small powered mowing machine, calculation method of stiff shaft dynamic at the time of movement.

\section{Discussion:-}

During the study process of the dynamic issues the optimal variants of theconstruction and validity of manual motorized mowing machine actually during the security working process of the small mechanized technical means is meant letting off, stopping, applying brakes during the work process and attention forms the dynamic process. During the letting process of the mechanism during the dynamic loading the quick braking of the machine which depends on rational calculation of the machine-details work and manual small mechanized mean becomes technologically heavy one and according to the work becomes defective and if during this time the turning moment become not enough the engine stops because the resistance value exceeds the stated norms.

Let's discuss the climbing moment of the stiff shaft in the free state when there is no any mowing process and its kinetics are interpreted as two circle mechanism ( picture 1)

If we will use for this mechanism the lagrange method then the second rank quality receives the following face ( $\mathrm{R}$. Makharoblidze . 1991)

$$
\frac{d}{d t}\left(\frac{\partial t}{\partial q}\right)-\frac{\partial t}{\partial q_{i}}+\frac{\partial \Pi}{\partial q_{i}}+\frac{d \Phi}{\partial q_{i}}=Q
$$


MWhere $\mathbf{T}$ and $\mathbf{M}$ represents the total kinetic and potential energy of the system.

$\Phi$ - spreading function of the energy

$\mathrm{Q}$ - moveming power

If you look we recieve the first picture:

$$
I_{(\varphi)} \frac{d \omega}{d t}+\frac{\omega}{2} \frac{d I_{(\alpha)}}{d t}=M_{g}(\omega)-M_{g}(\varphi, \omega, t)
$$

Where $\omega$ - represents moving corner speed.

$M_{g}(\omega)$-engine starting moment ( mechanical characteristics of the engine )

$M_{g}(\varphi, \omega, t)$ - separate meanings of the resistance moment

$t$ - time

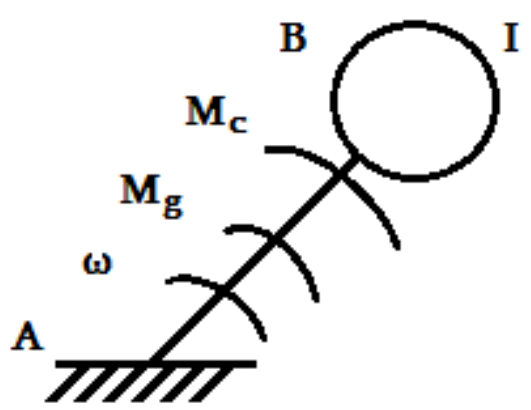

If we foresee the stiff shaft and cutting device then the second formula can be written inthe following way ( $\mathrm{M}$. Mamuladze, F. Abuselidze, 2009)

$$
\left\{\begin{array}{l}
I_{1} \frac{d^{2} \varphi_{1}}{d t^{2}}+C\left(\varphi_{1}-\varphi_{2}\right)=M_{g} \\
I_{2} \frac{d^{2} \varphi_{2}}{d t^{2}}+C\left(\varphi_{1}-\varphi_{2}\right)=-M_{c}
\end{array}\right.
$$

Where $\varphi_{1}$ and $\varphi_{2}$ - represents $I_{1}$ and $I_{2}$ the mass rotating angle $\mathrm{C}$ - moving shaft roughness

In the (3) formula including the deviation moment $M=C\left(\varphi_{1}-\varphi_{2}\right)$ gives the following type:

$$
\frac{d^{2} M}{d t^{2}}+\omega^{2} M=\frac{C M_{g}}{I_{1}}
$$

When the machine and work body is freed simultaneusly then $t=0, \varphi_{1}=\varphi_{2}=0, \frac{d \varphi_{1}}{d t}=\frac{d \varphi_{2}}{d t}=0$ the (3) formula can be written following way:

$$
M=\frac{C M_{g}}{I_{1}} \frac{1}{P^{2}+\omega^{2}}
$$

By calculating the 3rd formula's deviation moment can be written in the following way:

$$
M=\frac{M_{g} I_{2}}{I_{1}+I_{2}}\left(1-\cos \sqrt{\frac{C\left(I_{1}+I_{2}\right)}{I_{1} \cdot I_{2}} \cdot t}\right)
$$

when

$$
t_{\max }=\frac{\pi}{\sqrt{\frac{C\left(I_{1}+I_{2}\right)}{I_{1} \cdot I_{2}}}}(7)
$$

Maximal deviation moment is determined according to the following formula:

$$
M_{\max }=\frac{2 M_{g} I_{2}}{I_{1} \cdot I_{2}}
$$

or

$$
\frac{M_{\max }}{M_{g}}=\frac{2 \frac{I_{1}}{I_{2}}}{1+\frac{I_{2}}{I_{1}}}
$$

If the engine working inertia moment ratio is connected to the dynamic tension of themachine movement we recieve the following one: 


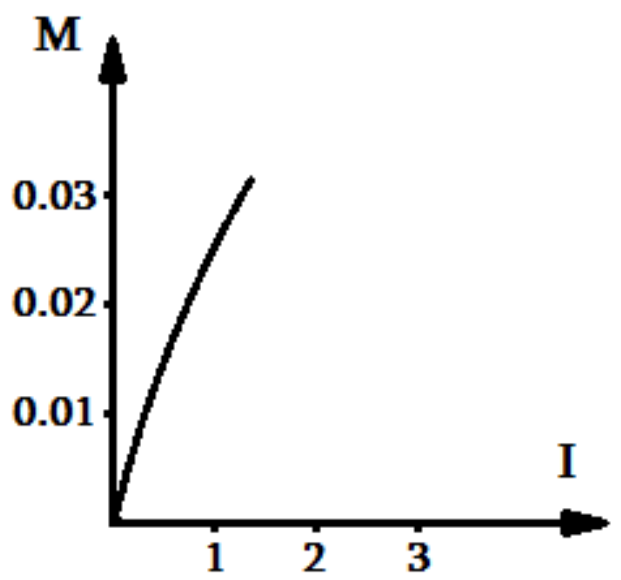

all of this shows that more is the turning moment of the working body the larger is the dynamic tension.

Let's us give the example: when between the working body and engine is located adherence machanism then 3rd formula is represented the following way:

$$
t=0, \quad \varphi_{1}=\varphi_{2}=0, \quad \frac{d \varphi_{2}}{d t}=0, \quad \frac{d M}{d t}=G \omega_{0}
$$

And the differencial equation can be written in the following way: (2)

From which

$$
M=G \omega_{0} \frac{P^{2}}{P^{2}+\omega^{2}}-\frac{C M_{g}}{I_{1}} \cdot \frac{1}{P^{2}+\omega^{2}}
$$

$$
\begin{gathered}
M(t)=\sqrt{\frac{C I_{1} I_{2}}{I_{1}+I_{2}}} \omega_{0} \cdot \sin \sqrt{\frac{C\left(I_{1}+I_{2}\right)}{I_{1}+I_{2}} \cdot t+\frac{M_{g} I_{2}}{I_{1}+I_{2}}\left(1-\cos \sqrt{\frac{C\left(I_{1}+I_{2}\right)}{I_{1} \cdot I_{2}} \cdot t}\right)} \\
z \cos \varphi_{0}=\omega_{0} \sqrt{\frac{C I_{1} I_{2}}{I_{1}+I_{2}}} \cdot z \sin \varphi_{0}=\frac{M_{g} I}{I_{1}+I_{2}} \\
z=\sqrt{\frac{\omega_{0}^{2} C I_{1} I_{2}}{I_{1}+I_{2}}-\frac{M_{g} I_{2}^{2}}{\left(I_{1}+I_{2}\right)^{2}}} \\
M_{(t)}=\frac{M_{g} I_{2}}{I_{1} \cdot I_{2}}+\sqrt{\frac{\omega_{0}^{2} C I_{1} I_{2}}{I_{1}+I_{2}}-\frac{M_{g} I_{2}^{2}}{\left(I_{1}+I_{2}\right)^{2}}} \sin \left(\omega t-\varphi_{0}\right) \\
\operatorname{tg} \varphi_{0}=\frac{M_{g} I_{2}}{\omega_{0} \sqrt{C I_{1} I_{2}\left(I_{1}+I_{2}\right)}} \\
\omega=\sqrt{\frac{C\left(I_{1}+I_{2}\right)}{I_{1} \cdot I_{2}}}
\end{gathered}
$$

Deviation moment reach the maximal level when

$$
\begin{gathered}
\omega t-\varphi_{0}=\frac{\pi}{2} \\
t_{\text {max }}=\frac{\pi+2 \varphi_{0}}{2 \omega} \\
M_{\text {max }}=\frac{M_{g} I_{2}}{I_{1}+I_{2}}+\sqrt{\frac{\omega_{0}^{2} C I_{1} I_{2}}{I_{1}+I_{2}}-\frac{M_{g}^{2} I_{2}^{2}}{\left(I_{1}+I_{2}\right)^{2}}}
\end{gathered}
$$

Or

$$
M_{\text {max }}=\frac{M_{g} I_{2}}{I_{1}+I_{2}}+\left[1+\sqrt{1+\frac{\omega_{0}^{2} C I_{1}\left(I_{1}+I_{2}\right)}{M_{g}^{2} I_{2}}}\right]
$$


When $\omega_{0}=0, C=0$, we recieve the (8) formula.

Let's represent all 18 formula in non dimension form that gives the following:

$$
\frac{M_{\max }}{M_{g}}=\frac{\rho}{\rho+1}\left[1+\sqrt{1+\frac{\omega_{0}^{2} C I}{M_{g}^{2}} \cdot \frac{\rho+1}{\rho}}\right]
$$

where:

$$
\rho=\frac{I_{2}}{I_{1}}
$$

(17) formula gives chance to determine the roughness of the manual motorized mowing machine $\mathrm{C}$ and deviation moment $\mathrm{M}$ by which we recieve:

$$
C=\frac{1}{\omega_{0}^{2} I_{1}}\left[\frac{I_{1}+I_{2}}{I_{2}}(M)^{2}-2 M_{g}(M)\right]
$$

Optimal parameters selection ability are interpreted accordingly. Actually from 19th and 20th formula is seen that deviation improves twice teh dynamic loading so the dynamic coeficient must be near 2 :

$$
k=1+\sqrt{1+\frac{\omega_{0}^{2} C I}{M_{g}^{2}} \cdot \frac{\rho+1}{\rho}}=2,1 .
$$

That's why

$$
\begin{gathered}
\frac{\omega_{0}^{2} C}{M_{g}^{2}}=0,2 \cdot \frac{\rho}{\rho+1} \\
C=0,21 \cdot \frac{M_{g}^{2}}{\omega_{0}^{2} I_{1}} \cdot \frac{\rho}{\rho+1}
\end{gathered}
$$

Reserach has been done on the basis of the technical data of the „Tiger Electronics“ engine:

$I_{1}=0,2 n / m^{2} ; I_{2}=0,04 n / m^{2} ; \omega=732 \sec ^{-1}[1] ; M_{g}=179 n$.

$\frac{I_{2}}{I_{1}}=\frac{0,04}{0,2}=0,2$.

then:

$C=0,04 \cdot \frac{M_{g}^{2}}{\omega_{0}^{2} I_{1}}=0,04 \frac{179^{2}}{732^{2} \cdot 0,2}=0,2 ;$

And

$t_{\text {max }}=\frac{\pi}{\sqrt{\frac{C\left(I_{1}+I_{2}\right)}{I_{1} \cdot I_{2}}}} \frac{3,14}{\sqrt{\frac{0,2(0,2+0,04)}{0,2 \cdot 0,04}}}=\frac{3,14}{\sqrt{\frac{0,2}{0,008}}}=1,3(\mathrm{sec}) ;$

$M_{\max }=\frac{2 M_{g} \frac{I_{1}}{I_{2}}}{1+\frac{I_{2}}{I_{1}}}=\frac{2 \cdot 179 \frac{0,2}{0,04}}{1+\frac{0,04}{0,2}}=6100(n)$.

\section{Conclusion:-}

Has been calculated manual motorized mowing machine, stiff shaft and segmented rotational device at the moment of movement during dynamic loading moment. Has been calculated the roughness value $\mathrm{C}=0.2$, maximal time $\mathrm{t}_{\max }=1.3 \mathrm{sec}$, maximal deviation moment $\mathrm{M}_{\max }=6100 \mathrm{n}$, actually was established that less the maximal deviation moment less is dynamic loading on the stiff shaft which largely depends on mining conditions, correct secure work of the working body and qualitative and lackless processes of the mowing technology.

\section{Literature:-}

1. M. Mamuladze, F. Abuselidze-Manual Motorized Mowing Machine with Dynamic Rotational Working Body (scientific work, journal „Moambe“, edition 24, Tbilisi, 2009)

2. R. Makharoblidze-Dynamic Process Optimization of the Agricultural Machines, Moscow, 1991

3. Ken Ohta, Yuji Ohgi, Kazuhiro Shibuya - Dealing with Stiffness: Shaft Dynamics in the Golf Swing - Journal of Sound and Vibration, 22 April, 1999

4. A.J Mazzei Jr, A. Argento, R.A. Scott- DYNAMIC STABILITY OF A ROTATING SHAFT DRIVEN THROUGH A UNIVERSAL JOINT-Journal of Sound and Vibration (Elseiver), Volume 222, Issue 1, 22 April 1999. 
5. T. C. Gmür, J. D. Rodrigues - Shaft Finite Elements for Rotor Dynamics Analysis- Journal of Vibration and Acoustics | Volume 113 | Issue 4 |1991.

6. J. Yang, T. C. Lim- Influence of Propeller Shaft Bending Vibration on Drivetrain Gear Dynamics - International Journal of Automotive Technology, Volume 16, Issue 1, USA 2015. 